\title{
Reducing the impact of source brightness fluctuations on spectra obtained by Fourier-transform spectrometry
}

\author{
Gretchen Keppel-Aleks, ${ }^{1, *}$ Geoffrey C. Toon, ${ }^{2}$ Paul O. Wennberg, ${ }^{1}$ and Nicholas M. Deutscher ${ }^{3}$ \\ ${ }^{1}$ California Institute of Technology, 1200 E. California Boulevard, Pasadena, California 91125, USA \\ ${ }^{2}$ NASA Jet Propulsion Laboratory, California Institute of Technology, 4800 Oak Grove Drive, Pasadena, \\ California 91109, USA \\ ${ }^{3}$ University of Wollongong, Wollongong, New South Wales 2522, Australia \\ ${ }^{*}$ Corresponding author: gka@gps.caltech.edu
}

Received 29 January 2007; revised 19 March 2007; accepted 22 March 2007;

posted 23 March 2007 (Doc. ID 79475); published 6 July 2007

\begin{abstract}
We present a method to reduce the impact of source brightness fluctuations (SBFs) on spectra recorded by Fourier-transform spectrometry (FTS). Interferograms are recorded without AC coupling of the detector signal (DC mode). The SBF are determined by low-pass filtering of the DC interferograms, which are then reweighted by the low-pass, smoothed signal. Atmospheric solar absorption interferograms recorded in DC mode have been processed with and without this technique, and we demonstrate its efficacy in producing more consistent retrievals of atmospheric composition. We show that the reweighting algorithm improves retrievals from interferograms subject to both gray and nongray intensity fluctuations, making the algorithm applicable to atmospheric data contaminated by significant amounts of aerosol or cloud cover. (C) 2007 Optical Society of America

OCIS codes: $\quad 010.1280,070.6020$.
\end{abstract}

\section{Introduction}

Traditionally, a high-pass analog filter has been used in Fourier-transform spectrometry (FTS) signal chains. This method, called AC recording, has been preferred over DC recording (in which the detector signal is not high-pass filtered prior to digitization) for two reasons. First, most analog-to-digital converters (ADCs) encompass positive and negative values, so recentering the interferogram at zero, as done in AC recording, enables the full dynamic range of the $\mathrm{ADC}$ to be utilized without the complication of subtracting an electrical offset. Second, AC recording facilitates gain switching, necessary in highresolution FTS to resolve the interferogram at both high and low optical path difference (OPD). At low OPD (the center burst of the interferogram) the fringes are several orders of magnitude larger in amplitude than those at high OPD. The use of a single gain to record the interferogram requires that the signal be sampled with very large dynamic

0003-6935/07/214774-06\$15.00/0

(C) 2007 Optical Society of America range, in excess of the 18 bits available from fast externally triggered ADCs. Therefore, gain switching during a scan is employed on many highresolution FTS instruments to avoid saturation of the interferogram at low OPD, while amplifying the small fringes at high OPD sufficiently to avoid digitization noise. To accomplish this, the large DC term must be eliminated to center the interferogram at $0 \mathrm{~V}$, consistent with the usual bipolar $\mathrm{ADC}$ inputs.

AC recording entails loss of information regarding source brightness fluctuations (SBFs) that occur during interferogram acquisition. Since SBFs are multiplicative, their effect cannot be removed simply by high-pass filtering; the high-frequency interferogram fringes have been amplitude modulated. Spectrometrists have long recognized that "ratio recording" reduces the impact of variations in source intensity, and have gone to great lengths to implement ratio-recording techniques (e.g., Ahlers et al., 1954 [1]). FTS systems are even more sensitive to such variations than are grating spectrometers due to the fact that the low and high resolution information is not acquired simultaneously. Because 
the zero path difference (ZPD) region of the interferogram sets the continuum level of the resulting spectrum, while the high OPD fringe amplitudes determine the line depths, SBFs during sampling of the interferogram affect broad and narrow spectral features differently. Intensity loss at one region of the interferogram can therefore distort the fractional line depths, since SBFs lead to multiplicative modulation of fringe amplitude.

The DC interferogram can be used to correct for SBFs, minimizing the errors that such fluctuations introduce into the spectra. With the use of an appropriate digital filter, the low-frequency variation due to SBFs can be separated from the higher frequencies that contain the interferometrically modulated energy. Knowledge of this DC intensity can then be used to correct for slow variations in source intensity as an interferogram is obtained. This is a fundamentally different approach than that of Shao et al., who attempt to identify and reject interferograms affected by SBFs [2].

We present here a method and an algorithm that correct for SBFs. DC interferograms are passed through a low-pass filter, and the resulting lowpass signal is used to normalize all regions of the interferogram. In reweighting the interferogram, the relative line depth to continuum level ratio is corrected as the fringes altered by SBFs are restored to their correct amplitude. The use of inexpensive high-dynamic range $\delta-\sigma$ ADCs (24 bits) facilitates such a correction. The large dynamic range eliminates the need for gain switching to properly resolve the high OPD regions of the interferogram. A single interferogram can then be recorded in low gain without high-pass filtering. Although not demonstrated here, similar benefits could be derived from a lower resolution ADC with two output channels: a low gain DC interferogram and a high gain AC interferogram. The low-gain, smoothed DC term would be sufficiently resolved to reweight an interferogram derived from the two channels.

In Section 2, we present our method for correcting DC interferograms and our reweighting algorithm. In Sections 3 and 4, we discuss the application of the reweighting algorithm to solar absorption spectrometry and present a comparison between data processed with and without the reweighting algorithm. Section 5 examines the efficacy of the reweighting algorithm in processing spectra affected by nongray SBFs. The influence of detector nonideality on the reweighted interferogram is discussed in Section 6.

\section{Methods}

The algorithm devised to correct for intensity variations is straightforward and had been suggested in the past by Brault (1985) [3]. We take a real-tocomplex fast Fourier transform (FFT) of the raw DC interferogram. To the resulting spectrum, we apply the spectral filter of Eq. (1), which retains frequencies below the cutoff frequency $s$ :

$$
F=\left\{\begin{array}{ll}
\left(\frac{1+\cos (\pi v / s)}{2}\right)^{N}, & \text { if } v<s \\
0, & \text { if } v>s
\end{array} .\right.
$$

We choose $s=300 \mathrm{~cm}^{-1}\left(180 \mathrm{~s}^{-1}\right)$ corresponding to $8 \%$ of the low-frequency detection limit for the spectra described here, and $N=8$. The frequency cutoff, $s$, should be minimized such that it is smaller than the lowest frequency at which interferometric modulation is measured, while still larger than the characteristic SBF frequency. The parameter $N$ determines the steepness of the filter cutoff. A large difference between the characteristic SBF frequency and the low-frequency detection limit allows a wider range of permissible $s$ values and lowers the sensitivity to the choice of $N$. In instances where the highest SBF frequency and the low-frequency detection limit are similar, a higher value of $N$ is desired to increase the filter steepness. We discuss the sensitivity of the data to choice of $s$ in Section 6 .

We take the inverse Fourier transform of the lowfrequency spectrum that results from Eq. (1) using a complex-to-real routine. The resulting low-passfiltered interferogram provides a measure of the $\mathrm{SBF}$ at frequencies well below the signal due to interferometric modulation. Ratioing the raw interferogram to this smoothed interferogram yields an interferogram with a constant DC level of 1 , with all regions of the interferogram having a uniform intensity weighting;

$$
I_{\text {corr }}(x)=\frac{I_{\text {raw }}(x)}{I_{\text {smooth }}(x)} * I_{\text {smooth }}(\mathrm{ZPD})
$$

We choose to scale the reweighted interferogram by the DC level at ZPD, as written in Eq. (2), so that each interferogram retains information on the absolute intensity at ZPD, which is helpful in many applications. The corrected interferogram can then be processed using standard interferogram processing routines.

\section{Application}

SBFs affect many spectra obtained by FTS. Remote sensing is particularly susceptible as scene brightness often changes during the acquisition of an interferogram. We illustrate the benefits of the reweighting algorithm with measurements of infrared solar spectra obtained from the Earth's surface. Ground-based direct solar absorption spectrometry is a common technique used to infer atmospheric composition. High resolution FTS has been used historically by the Network for the Detection of Atmospheric Composition Change (previously called the Network for the Detection of Stratospheric Change [4]). Although using the Sun as a source provides an excellent signal-tonoise ratio (SNR), the source intensity can vary on 
the time scale of a single scan due to attenuation by clouds and aerosol. Such variability degrades the accuracy and precision of gas amounts retrieved from such spectra.

In our application, we use near-infrared solar absorption spectra to obtain total column abundance of atmospheric gases, including $\mathrm{O}_{2}, \mathrm{CO}_{2}, \mathrm{CO}, \mathrm{CH}_{4}$, and $\mathrm{H}_{2} \mathrm{O}$ [5]. These measurements are obtained at Park Falls, Wisconsin and Darwin, Australia, sites affiliated with the Total Carbon Column Observing Network (TCCON) [6]. The goal of TCCON is to provide constraints on variability in surface fluxes of $\mathrm{CO}_{2}$. To accomplish this, it is necessary to obtain precisions in column retrievals better than $0.5 \%$. To meet the required precision without the DC correction, interferograms obtained with high SBF, as determined by a measurement of the total solar intensity on a solar tracker quadrant sensor, were discarded. Eliminating these spectra greatly reduced the range of atmospheric conditions under which worthwhile observation was possible. Even optically thin clouds crossing the field of view of the instrument rendered the measurements unusable. Incorporation of the DC correction allows many of these otherwise-discarded spectra to be used.

Data are obtained using a Bruker IFS 125HR Fourier-transform spectrometer. The instrument is optimized for recording in the near-infrared, with

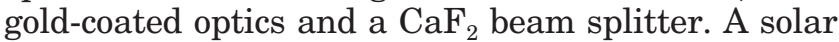
tracker with a silicon quadrant sensor centers the solar image on the entrance fieldstop of the FTS. We record the solar intensity at the quadrant sensor at a rate of $0.5 \mathrm{~Hz}$ over the $90 \mathrm{~s}$ duration of a single-sided scan. The ratio of the standard deviation to the mean of the solar intensity represents the solar intensity variation. If the variation measured by the quadrant sensor for a given interferogram falls below a threshold, we transform the interferogram and retrieve gas columns from the spectrum. Interferograms are recorded on two detectors simultaneously. We obtain full spectral coverage between 3800 and $15,750 \mathrm{~cm}^{-1}$ using an InGaAs detector $\left(3800-11,000 \mathrm{~cm}^{-1}\right)$, and a Si diode detector $\left(10,000-15,750 \mathrm{~cm}^{-1}\right)$. The detectors are coupled to a 24 bit $\delta-\sigma$ ADC. We obtain direct solar absorption spectra at $0.02 \mathrm{~cm}^{-1}$ resolution (45 $\mathrm{cm}$ OPD) and a scan velocity of $10 \mathrm{kHz}$. For more information on the experimental setup and analysis procedure, see Washenfelder et al. (2006) [5].

\section{Results}

Figure 1(a) shows an interferogram obtained in DC mode on the InGaAs detector. The smoothed, lowpass signal is shown in Fig. 1(b). This interferogram was obtained with solar intensity variation of $14 \%$; since intensity decreased during the scan, the weight of the high OPD regions of the corrected interferogram, shown in Fig. 1(c), has been increased, amplifying those fringes and restoring the spectral lines to their correct depths. The reweighted interferogram is then transformed using slice-ipp, an FFT algorithm developed at NASA's

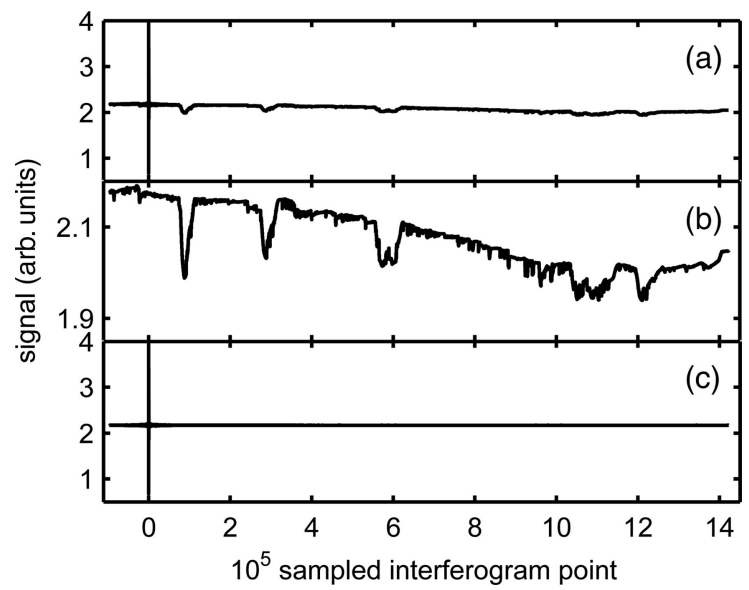

Fig. 1. (a) Raw DC interferogram, $I_{\text {raw }}$, with $14 \%$ solar intensity variation; (b) smoothed, low-pass signal, $I_{\text {smooth }}$; (c) corresponding reweighted interferogram, $I_{\text {corr }}$.

Jet Propulsion Laboratory (JPL), which phase corrects the interferogram prior to taking the real-tocomplex FFT. We use GFIT, a line-by-line nonlinear least-squares fitting program also developed at NASA's JPL to determine atmospheric composition from the spectra.

We test the algorithm by comparing atmospheric column amounts retrieved from spectra derived both from reweighted interferograms and from control DC interferograms that have not been reweighted. We use data from both cloudy and clear days to determine the effect of the algorithm on both high quality and potentially compromised data.

Retrievals from the spectra obtained from the reweighted interferograms show substantially increased precision compared with control data. Shown in Fig. 2 are the $\mathrm{CO}_{2}$ columns retrieved from FTS spectra measured at the U.S. Department of Energy Atmospheric Radiation Measurement site in Darwin, Australia (-12.4 S and 130.9 E) on 29 October 2005. This day was characterized by partly cloudy skies with solar intensity variations between $2 \%$ and $50 \%$ during individual scans. Since the site is coastal, we expect minimal diurnal variability in $\mathrm{CO}_{2}$. We retrieve near-constant gas columns from the reweighted data over the range of observed solar intensity variation, in contrast to the control data where the scatter in the retrieved columns becomes large at high solar variation. The inset to Fig. 2 shows that the reweighting algorithm reduces the scatter and gives more consistent retrievals even when solar intensity variations fall below $10 \%$. For the full data set of 227 control scans, the column median is 8.24 $\times 10^{21}$ molecules, with $75 \%$ of the retrieved columns falling between $-1.2 \%$ and $0.9 \%$ of the median value. In contrast, the reweighted data have a median column retrieval of $8.25 \times 10^{21}$ molecules $/ \mathrm{cm}^{2}$, with $75 \%$ of the data falling within $-0.3 \%$ and $0.4 \%$ of the median. For scans taken with solar intensity variation less than $10 \%$, the control data show a spread between $-0.6 \%$ and $0.7 \%$ of the median, while for the 


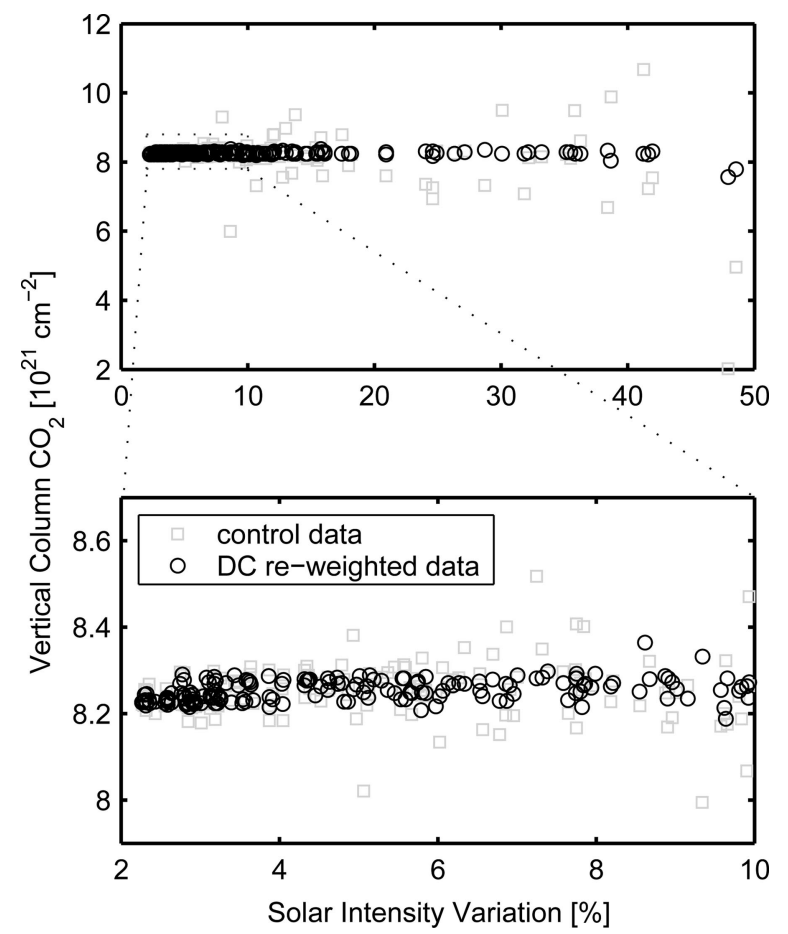

Fig. 2. Comparison between $\mathrm{CO}_{2}$ retrievals from reweighted (dark circles) and the raw control (light squares) spectra as a function of relative solar intensity variation during a $90 \mathrm{~s}$ scan. The lower panel zooms in on relative solar intensity variation below $10 \%$.

reweighted spectra, the spread is between $-0.03 \%$ and $0.4 \%$ of the median.

To test whether the reweighting algorithm introduces a bias to the gas retrievals, we use data obtained at Darwin when solar intensity variations were below $3 \%$ for all scans recorded. As shown in Fig. 3, we see a small negative bias in the reweighted data. For the 238 scans used from 19 December 2005, the mean difference is $-0.016 \%$, with a standard deviation of $0.048 \%$ and a standard error of the sampling distribution of $0.003 \%$, indicating a statistically significant negative bias in the reweighted data. This

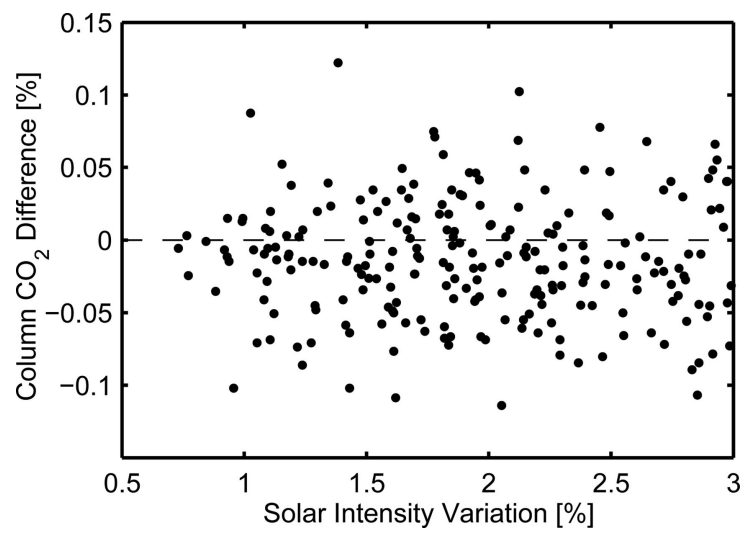

Fig. 3. Percent difference between $\mathrm{CO}_{2}$ retrievals from spectra obtained from reweighted and control interferograms with solar intensity variation less than $3 \%$. bias could be real; the gas columns determined in the control analysis could be higher than the true atmospheric column. We do not have perfect data completely unaffected by SBFs with which to test the algorithm, so we must use data with solar intensity variation less than $3 \%$ as a proxy. This bias could also be due to artifacts near ZPD in the smoothed interferogram, which will be discussed further in Section 6 . In either case, the $0.02 \%$ bias is quite small and is undetectable in observations made at solar intensity variation between $3 \%$ and $50 \%$. As seen in Fig. 3, the difference between the corrected and uncorrected $\mathrm{CO}_{2}$ column is generally less than $0.1 \%$, with the largest differences at solar intensity variation approaching $3 \%$. The data suggest that the algorithm improves scatter in the retrievals at all solar intensity variations, with its impact on retrievals increasing with solar intensity variation.

Based on the performance of the algorithm in improving retrievals, we have transformed the data obtained at Darwin from August 2005 to August 2006 using the DC reweighting algorithm. DC acquisition was first implemented in late October 2005, so that the first 2 months of data were obtained in AC mode using a high-pass filter. Figure 4 shows the $\mathrm{O}_{2}$ volume mixing ratio retrieved at solar intensity variations less than 20\%. Note the dramatic transition between the $\mathrm{AC}$ and $\mathrm{DC}$ regimes. Prior to implementing DC recording, we used a threshold of $2 \%$ solar intensity variation to obtain a high-precision record under AC acquisition. Using this threshold, we would have discarded all interferograms obtained on 85 out of 286 days between August 2005 and August 2006. Using a higher cutoff of $20 \%$ for DC data, the DC reweighting algorithm has enabled us to include data from those 85 days and improve the quality of the data from the other 201 days.

The SNR of the spectra decreases by approximately $20 \%$, from 840 to 660 near the peak of the InGaAs spectrum and from 390 to 320 near the peak of the $\mathrm{Si}$ spectrum, when we switch from $\mathrm{AC}$ to $\mathrm{DC}$ acquisi-

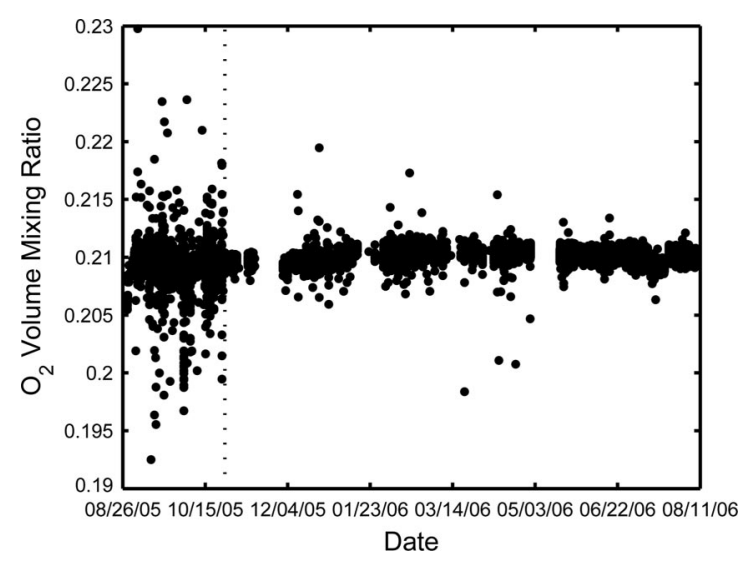

Fig. 4. Time series of the oxygen mixing ratio retrieved from spectra at Darwin, Australia. Prior to November 2005, AC data acquisition was used. Plotted here are data obtained between $20^{\circ}$ and $70^{\circ}$ solar zenith angles. Only spectra obtained with relative solar variation less than $20 \%$ are shown. 
tion. We stress that this reduction in SNR has a negligible effect on our total error budget compared with the errors introduced by uncorrected SBF. The substantial gain in precision and the increase in the range of atmospheric conditions in which measurements can be used both far outweigh this $20 \%$ reduction in SNR.

\section{Nongray Source Brightness Fluctuations}

The theory behind the SBF correction method described here is valid for gray source intensity fluctuations; if the shape of the spectrum remains unchanged, and only its intensity varies, the DC correction should be perfect. However, clouds and aerosol in the atmosphere scatter light preferentially at shorter wavelengths; SBFs are thus larger at shorter wavelength than at longer wavelength (i.e., nongray).

To examine the effect of nongray SBFs on our gas retrievals, we distort a sample of ideal interferograms with low relative solar intensity variations $(0.5 \%-3 \%)$ to simulate the effects of nongray intensity fluctuations. Although the effects of nongray attenuation could be investigated using a stable light source, we chose instead to model the effects of nongray SBFs, since no such lamp was available. We Fourier transform an ideal interferogram and use $S_{\text {distorted }}(v)=S_{\text {original }}(v) \exp \left[-\tau(v / 15,750)^{\alpha}\right]$ to distort the resulting spectrum for $v$ between 300 and 15,750 $\mathrm{cm}^{-1}$, increasing the value of $\tau$ to increase total attenuation [Fig. 5(a)]. We choose angstrom exponent $(\alpha)$ of 0.3 , a realistic value for clouds in the atmosphere. The ratio of the area under the distorted spectrum to that of the original spectrum between the frequency limits is used to scale the $v=0$ point of the distorted $\mathrm{DC}$ spectrum and the associated ringing at frequencies below $300 \mathrm{~cm}^{-1}$. The distorted spectra are inverse transformed; the resulting interferograms [Fig. $5(b)]$ have been influenced by time-independent, nongray attenuation.

We interpolate between the attenuated interferograms to create an interferogram that exhibits a temporal dependence to the modeled nongray attenuation [Fig. 5(c)]. This interferogram is then reweighted using the DC correction algorithm [Fig. $5(d)]$. The control and reweighted interferograms are transformed [Figures 5(e) and (f)], and gas columns are retrieved from each spectrum. We have simulated two forms of transience: total intensity linearly falling off by $50 \%$ at high OPD and total intensity linearly increasing by a factor of 2 from ZPD to maximum OPD.

We retrieve $\mathrm{CO}_{2}$ and $\mathrm{O}_{2}$ from two different bands to determine the performance of the $\mathrm{DC}$ reweighting algorithm. The nongray distortion introduces errors between $1 \%$ and $4 \%$ that are dependent on the characteristics of the fitting window and on the temporal distribution of the distortion. Table 1 shows the impact of these simulations on retrievals from the three bands: $\mathrm{CO}_{2} 6180-6260 \mathrm{~cm}^{-1}, \mathrm{O}_{2} 7765-8005 \mathrm{~cm}^{-1}$, and $\mathrm{O}_{2} 12,940-13,190 \mathrm{~cm}^{-1}$ ( $A$ band).

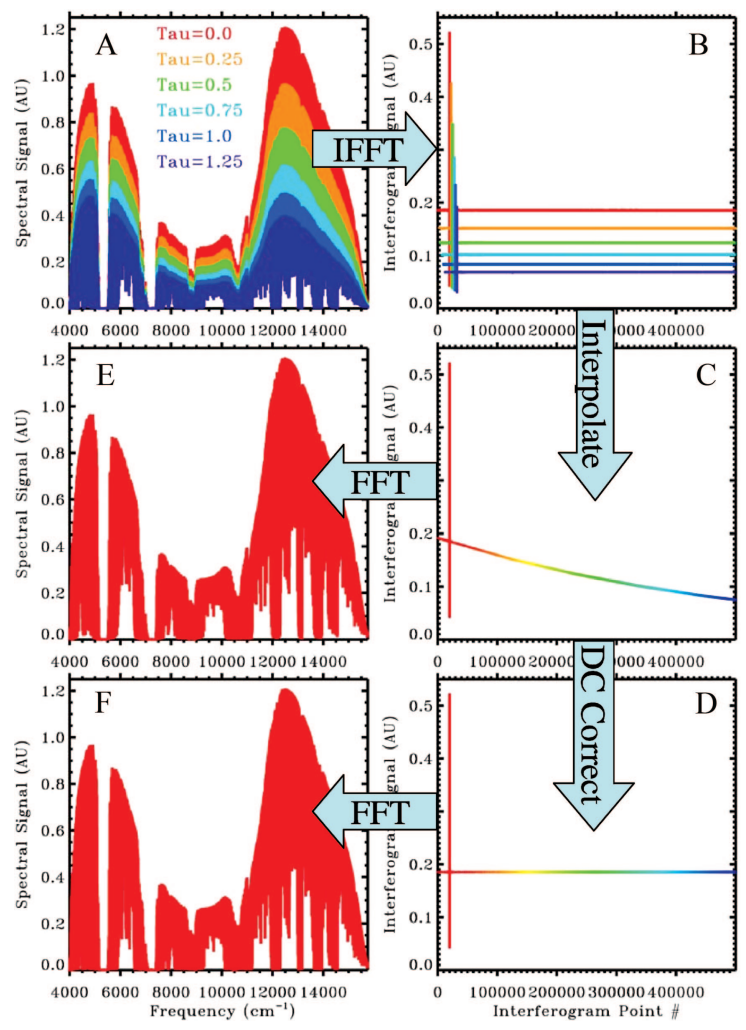

Fig. 5. Spectrum distorted with nongray interference of optical depth ranging from 0.0 (original spectrum) to 1.25 at $15,750 \mathrm{~cm}^{-1}$ (panel A). The spectra are inverse Fourier transformed to yield the interferograms in panel B. We interpolate between these interferograms to generate the interferogram in panel $\mathrm{C}$, which exhibits spectrally and temporally dependent attenuation compared with the original interferogram. The attenuated interferogram is reweighted using the DC correction to yield the interferogram in panel D. We then transform each interferogram to yield the control spectrum in panel $\mathrm{E}$ and the reweighted spectrum in panel $\mathrm{F}$. Although the spectra in $\mathrm{E}$ and $\mathrm{F}$ appear identical, the line depths in the uncorrected spectrum (E) are more shallow than those in panel F.

The distorted interferograms that have been reweighted using our algorithm have errors less than $0.4 \%$, in contrast to errors of several percent in data obtained without using the reweighting algorithm. These simulations demonstrate that the method is robust to nongray SBFs. An angstrom exponent of 0.3 is used in the simulations described in Table 1 , but we also further simulated SBFs with an angstrom exponent as large as 4.0 and still see significant improvements, with errors generally smaller than $0.2 \%$ in the column retrievals from reweighted interferograms. We therefore recommend use of this algorithm for DC-recorded data in all situations, even when the interference exhibits spectral dependence.

\section{Artifacts at Zero Path Difference}

The reweighting method is sensitive to nonideal detector behavior near ZPD [Fig. 6(a)]. In addition to choosing $s$ in Eq. (1) below the low-frequency detection limit, but above SBF frequency, the filter cutoff can be selected to minimize artifacts resulting from 
Table 1. Retrieval Errors [\%] from Spectra Distorted by Nongray SBF

\begin{tabular}{|c|c|c|c|c|}
\hline \multirow[b]{2}{*}{ Retrieved Species } & \multicolumn{2}{|c|}{ Low OPD Distortion } & \multicolumn{2}{|c|}{ High OPD Distortion } \\
\hline & (Control) & (Reweighted) & (Control) & (Reweighted) \\
\hline $\mathrm{CO}_{2}\left(6180-6260 \mathrm{~cm}^{-1}\right)$ & 4.185 & 0.081 & -1.902 & -0.017 \\
\hline $\mathrm{O}_{2}\left(7765-8005 \mathrm{~cm}^{-1}\right)$ & 3.264 & 0.368 & -1.442 & -0.084 \\
\hline $\mathrm{O}_{2}\left(12,940-13,190 \mathrm{~cm}^{-1}\right)$ & -0.549 & -0.012 & 0.216 & -0.004 \\
\hline
\end{tabular}

detector nonideality. When using a filter cutoff at $80 \%$ of our low spectral frequency limit $\left(3000 \mathrm{~cm}^{-1}\right)$, we observed a dip in the low-pass signal at ZPD [Fig. $6(b)]$. We attribute this dip to detector nonlinearity. Although not discussed further here, this dip can be used to diagnose, model, and account for nonlinearity in the detector. When the frequency cutoff is reduced to $8 \%$ of the low-frequency limit $\left(300 \mathrm{~cm}^{-1}\right)$, these artifacts are no longer visible, as demonstrated in Fig. 6(c). The presence of an artifact at ZPD in the smoothed interferograms can bias the resulting fits. The dips at ZPD amplify the center burst of the reweighted interferogram, raising continuum levels and inducing a negative bias in the retrievals from reweighted data, an order of magnitude larger than the $0.02 \%$ bias observed when $s$ is $8 \%$ of the lowfrequency detection limit. The detector at Darwin exhibits only a small nonlinearity; it is possible that for a less linear detector, choosing $s$ above the SBF frequencies would not sufficiently smooth away the artifact observed in Fig. 6(b). In such a case, the DC

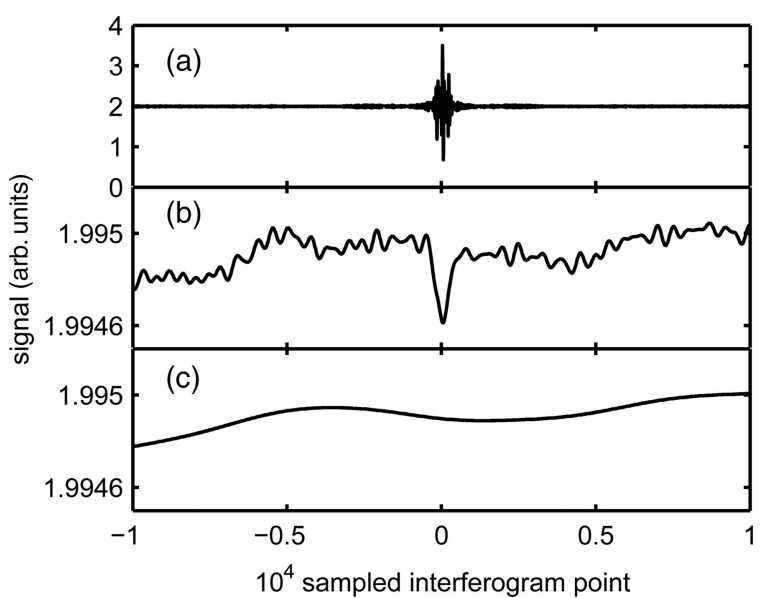

Fig. 6. (a) Center burst of raw interferogram. (b) Low-pass signal resulting from a filter with $s=3000 \mathrm{~cm}^{-1}$. The observed dip at ZPD is likely due to detector nonlinearity. (c) Low-pass interferogram resulting from filter with $s=300 \mathrm{~cm}^{-1}$. The dip at ZPD has been smoothed away in lowering the frequency cutoff. correction algorithm would need to be modified to correct for detector nonlinearity as well.

\section{Conclusion}

The influence of SBFs on spectra recorded using DC signal processing can be substantially reduced by a simple reweighting algorithm. We expect that this correction method will have an increasingly significant impact on choices made regarding $\mathrm{DC}$ versus $\mathrm{AC}$ interferogram acquisition, especially as higher resolution ADCs become more prominent in FTS. Even without high-resolution ADCs, DC recording of lowgain interferograms provides the essential tools for separating the low-frequency source variations from high-frequency interferometric modulation, enabling source brightness fluctuations to be corrected.

The authors thank D. Griffith and G. Bryant for their role in data acquisition at the Darwin FTS site. J.-F. Blavier is gratefully acknowledged for helpful discussions. This analysis was supported by a grant from NASA (NNGO5-GD07G). The Darwin FTS was developed for the OCO project at NASA JPL; G. Keppel-Aleks acknowledges support from the NSF Graduate Fellowship Program.

\section{References}

1. N. H. E. Ahlers and H. P. Freedman, "A simple ratio-recording spectrometer,” J. Sci. Instrum. 32, 61-64 (1955).

2. L. Shao, M. J. Pollard, P. R. Griffiths, D. T. Westermann, and D. L. Bjorneberg, "Rejection criteria for open-path Fouriertransform infrared spectrometry during continuous atmospheric monitoring," Vib. Spectrosc. 43, 75-85 (2007).

3. J. W. Brault, "Fourier-transform spectrometry," in High Resolution in Astronomy, A. O. Benz, M. C. E. Huber, and M. Mayor, eds. (Swiss Society of Astrophysics and Astronomy, 1985), pp. $3-62$.

4. M. J. Kurylo and S. Solomon, Network for the Detection of Stratospheric Change, NASA report, Code EEU (NASA, 1990).

5. R. A. Washenfelder, G. C. Toon, J.-F. Blavier, Z. Yang, N. T. Allen, P. O. Wennberg, S. A. Vay, D. M. Matross, and B. C. Daube, "Carbon dioxide column abundances at the Wisconsin Tall Tower site,” J. Geophys. Res. 111, D22305 (2006).

6. "The Total Carbon Column Observing Network," http://tccon. caltech.edu. 